\title{
Athina Markou's contributions to treatment development for mental illnesses: a perspective
}

\author{
Lois Winsky $^{1} \cdot$ Linda S. Brady $^{1}$
}

Received: 4 November 2016 / Accepted: 8 November 2016/Published online: 24 November 2016

(C) Springer-Verlag Berlin Heidelberg (outside the USA) 2016

\section{Understanding reward circuits}

Dr. Athina Markou received her Ph.D. from the University of California San Diego in 1991 and, very early in her career, she showed a strong skill for building strong research programs in substance use and psychiatric disorders by obtaining both pre-doctoral and postdoctoral Individual National Research Service Awards from the National Institute on Drug Abuse (NIDA). These first projects emphasized reward mechanisms in relation to drugs of abuse, a research area that she would continue to foster throughout her career. Athina received her first National Institutes of Health (NIH) Research Project Grant (R01) grant from NIDA in 2000, "Neurobiology of Nicotine Reward and Withdrawal". During this time, Athina co-authored a highly cited article on the construct validity of intracranial self-stimulation (ICSS) as a tool to elucidate the neurobiological basis of reward-related processes (Markou and Koob 1992). Her interests not only in reward processes but also in reward deficits in psychiatric disorders led to her second R01 award 2 years later, this time from the National Institute of Mental Health (NIMH, "Negative symptoms of schizophrenia: animal models"), focused on developing and validating paradigms that can be used to characterize constructs relevant to a cluster of negative symptoms of schizophrenia in experimental animals. In this project, she applied

Linda S. Brady

lbrady@mail.nih.gov

1 Division of Neuroscience and Basic Behavioral Neuroscience, National Institute of Mental Health, National Institutes of Health, Bethesda, MD 20892, USA
ICSS in rodents to evaluate gene expression changes within circuits mediating reward. In 2002, when the NIMH grant was awarded, it was quite unusual for investigators to employ model systems to interrogate domains of dysfunction in a manner that is consistent with the Research Domains Criteria (RDoC) initiative that was undertaken by NIMH a half a dozen years later (2009). Athina's research focus on reward processing had an impact on understanding reward deficits in psychiatric disorders (negative symptoms in schizophrenia) (Barnes et al. 2014) as well as substance use disorders by generating basic knowledge about circuits and molecular targets for the discovery and development of novel treatments for these disorders.

\section{Building collaborations to advance novel therapeutics}

Throughout her career, Athina served as a catalyst for building translational approaches in treatment development for psychiatric and substance use disorders and showed tremendous talent for bringing together groups of scientists to address research needs. In one such effort, Athina created a collaborative network to develop novel treatments for nicotine abuse and mental disorders. The group comprised experts in medicinal chemistry and drug development, from academia and pharma, to complement her significant expertise with in vivo measures and behavioral pharmacology. The project was supported through a joint NIMH and NIDA initiative, the National Cooperative Drug Discovery Group for the Treatment of Mood Disorders or Nicotine Addiction. Athina's project was one of three grants that were awarded in response to the NIH initiative. The cooperative agreement co-funded by NIMH and NIDA had the goal of developing gamma-aminobutyric 
acid $\mathrm{B}$ receptor $\left(\mathrm{GABA}_{\mathrm{B}}\right)$ positive allosteric modulators (PAMs) as antidepressants and/or aids to smoking cessation. Athina's group uncovered a wealth of potential applications of $\mathrm{GABA}_{\mathrm{B}}$ ligands, including attenuation of the rewarding properties of cocaine in $\mathrm{GABA}_{\mathrm{B}}$ PAM-treated rats and stressreducing effects of $\mathrm{GABA}_{\mathrm{B}}$ antagonists (Slattery et al. 2005). A selective $G_{A B A_{B}}$ PAM BHF177 was distinguished from both a $\mathrm{GABA}_{\mathrm{B}}$ agonist and a benzodiazepine anxiolytic in several experimental paradigms on stress- and anxietyrelated behaviors in mice (Li et al. 2013). BHF177 was found to be ineffective across a wide range of anxiety tests in mice. In a later study looking at the effects of $\mathrm{GABA}_{\mathrm{B}}$ PAMs in startle-based anxiety tests (light- and fear-potentiated startle) in rodents, BHF177 was found to have anxiolytic-like effects on light-enhanced startle only in rats that exhibit a high anxiety state (Der-Avakian et al. 2016). Athina and her group concluded from these studies that the disparate findings across stress- and anxiety-related paradigms in rodents reflected the differences in the underlying neurobiology of the anxiety constructs and species-specific pharmacology of $\mathrm{GABA}_{\mathrm{B}}$ PAMs. This theme would recur in Athina's later efforts to build translational, cross-species measures as assays for therapeutic development.

While Athina maintained cooperative agreement awards from NIDA and NIAAA to advance new treatments of substance use disorders, her efforts in treatment development for mental illnesses shifted towards developing innovative ligands for metabotropic glutamate receptor subtype 2- and -3 (mGluR2/3) as novel treatments for mood disorders (Dhanya et al. 2014; Grivas et al. 2013; Pitsikas and Markou 2014). This interest lead to an NIMH-funded project co-led with Nicholas Cosford at Sanford Burnham Prebys Medical Discovery Institute to optimize the potency of group II mGluR antagonists and negative allosteric modulators for activity in experimental paradigms that tap into domains of anhedonia relevant to depression. Beyond this, Athina contributed her expertise to many additional groups interested in treatment development for psychiatric and substance use disorders and the development and validation of translational assays to assess reward deficits in animals and humans.

\section{Advancing innovative translational assays to improve treatment development for mental disorders}

In 2012, Athina co-authored a highly cited review focused on the neural circuits underlying anhedonia and other rewardrelated deficits in psychiatric disorders, i.e., avolition, deficits in anticipation/prediction, valuation, reinforcement learning, and decision-making (Der-Avakian and Markou 2012). She stressed the need to understand which of these rewardrelated processes, and the underlying circuits, are disrupted in individuals with psychiatric disorders in order to develop treatments targeted to the specific reward deficits. She emphasized in recent reviews (Der-Avakian et al. 2016; Der-Avakian et al. 2013) the need for translational measures of rewardrelated processes that can be used in rodents and humans and laid out important considerations for cross-species behavior assessments of reward and motivation.

One of Athina's most recent efforts involved contributing to the construction of a research team aimed at identifying and evaluating translational brain physiological measures in order to improve the potential for preclinical animal data to predict effects of novel pharmacologic or circuit-based interventions in humans (Novel Assays to Address Translational Gaps in Treatment Development). Athina was a critical contributor and key driver to the collaboration with Diego Pizzagalli and his team of highly skilled researchers at McLean Hospital along with her group at the University of California San Diego, "Novel Cross-Species Neurophysiological Assays of Reward and Cognitive Domains". The NIMH-funded cooperative agreement project aims to explore electrophysiological (EEG) signatures during rewarding tasks (probabilistic reward, flanker, 4-choice serial reaction time, and a probabilistic reversal learning task) in both healthy rats and humans to assess their potential validity as translational assays for developing and advancing novel interventions for individuals with reward deficits in drug discovery and early stage clinical trials.

Athina Markou's many contributions to neuroscience and therapeutic discovery will continue to have a significant impact for understanding mechanisms underlying the variations of reward deficits seen across diagnoses for psychiatric and substance use disorders.

\section{References}

Barnes SA, Der-Avakian A, Markou A (2014) Anhedonia, avolition, and anticipatory deficits: assessments in animals with relevance to the negative symptoms of schizophrenia. Eur Neuropsychopharmacol 24:744-758

Der-Avakian A, Markou A (2012) The neurobiology of anhedonia and other reward-related deficits. Trends Neurosci 35:68-77

Der-Avakian A, D'Souza MS, Pizzagalli DA, Markou A (2013) Assessment of reward responsiveness in the response bias probabilistic reward task in rats: implications for cross-species translational research. Transl Psychiatry 3:e297

Der-Avakian A, Barnes SA, Markou A, Pizzagalli DA (2016) Translational assessment of reward and motivational deficits in psychiatric disorders. Curr Top Behav Neurosci 28:231-262

Dhanya RP, Sheffler DJ, Dahl R, Davis M, Lee PS, Yang L, Nickols HH, Cho HP, Smith LH, D'Souza MS, Conn PJ, Der-Avakian A, Markou A, Cosford ND (2014) Design and synthesis of systemically active metabotropic glutamate subtype- 2 and -3 (mGlu2/3) receptor positive allosteric modulators (PAMs): pharmacological characterization and assessment in a rat model of cocaine dependence. J Med Chem $57: 4154-4172$ 
Grivas V, Markou A, Pitsikas N (2013) The metabotropic glutamate 2/3 receptor agonist LY379268 induces anxiety-like behavior at the highest dose tested in two rat models of anxiety. Eur J Pharmacol 715:105-110

Li X, Risbrough VB, Cates-Gatto C, Kaczanowska K, Finn MG, Roberts AJ, Markou A (2013) Comparison of the effects of the $\mathrm{GABA}_{B}$ receptor positive modulator BHF177 and the GABAB receptor agonist baclofen on anxiety-like behavior, learning, and memory in mice. Neuropharmacology 70:156-167

Markou A, Koob GF (1992) Construct validity of a self-stimulation threshold paradigm: effects of reward and performance manipulations. Physiol Behav 51:111-119
Pitsikas N, Markou A (2014) The metabotropic glutamate 2/3 receptor agonist LY379268 counteracted ketamine-and apomorphine-induced performance deficits in the object recognition task, but not object location task, in rats. Neuropharmacology 85:27-35

Slattery DA, Markou A, Froestl W, Cryan JF (2005) The GABAB receptor-positive modulator GS39783 and the GABAB receptor agonist baclofen attenuate the reward-facilitating effects of cocaine: intracranial self-stimulation studies in the rat. Neuropsychopharmacology 30:2065-2072 